\title{
CPG Design in Bipedal Locomotion by Machine Learning Techniques: A Review
}

\author{
Rajeev Kumar \\ Ph.D Scholar, Dr. APJ Abdul \\ Kalam Technical University \\ Lucknow, U.P
}

\author{
Laxman Singh \\ Department of ECE, \\ Noida Institute of Engg., \& \\ Technology, Greater Noida
}

\author{
Rajdev Tiwari \\ Department of Computer science \\ \& Engg \\ GNIOT, Greater Noida, Uttar \\ Pradesh
}

\begin{abstract}
The natural species found in this world exhibit some form of intelligence in their conducts. The human being is the ultimate benchmark among all the species, which shows the intelligence in almost every conduct in their life. So in the world of artificial intelligence we are trying to mimic the behavior of the human being through the machines. The artificial counterpart of the human being who has the resemblance with the human being is called biped or humanoid. In this paper we review the machine learning techniques which are popular among researchers from last 10 to 15 years for learning task in robotics. Machine learning techniques specifically includes supervised learning, unsupervised learning and Reinforcement learning.
\end{abstract}

\section{Keywords}

Biped, Central pattern generator (CPG), Deep Learning, Machine Learning, neural oscillators.

\section{INTRODUCTION}

The efficient movement in the typical environments is the key property of human beings and other species exist in the real world. The ability of reproduction, the search of food, the ability to move from one place to another obviously requires the movement capabilities and the nervous system exist in human beings to drive and control all these aspects [1]. If we think about to design a machine that exhibit the functionalities shown by human beings, obviously it is typical to achieve all these functionalities, but maximum efforts should be expected from the researchers and scientists.

The field of biology and humanoid robotics is highly interrelated with one another in the sense that the locomotion behavior of the human beings is adopted in humanoids. The researchers work on the control mechanism of human body mimicked in humanoids. But now humanoids provide some techniques back to human beings to test some biological hypothesis [2].
From the existing evidences it is assumed that vertebrate locomotion is controlled by a central pattern generator (CPG) capable of producing the different types of rhythms associated with different types of gaits. It is often assumed that the Central pattern generators are built by using the oscillators of various types [3]. The different types of oscillators are Rayleigh oscillator, Vanderpol oscillator and the most often used is Matsuoka neural oscillator; through these the modeling of CPGs are done and described by the identical systems of differential equations [4].

The paper is organized as follows: In the second section, we discussed about various mathematical models of biological CPGs developed by different researchers. Third section presents the review of the CPG models specially designed and developed for biped robots. Fourth section focus on the techniques to design CPG models for specific tasks and some learning aspects using neural networks. Finally in the last section, we discuss the challenges for the humanoid research, and growth of machine learning techniques for humanoid robots.

\section{MATHEMATICAL MODELS OF CENTRAL PATTERN GENERATOR}

Researcher proposed a number of models for the locomotion of humanoids as well as for animals. Some animals may be two legged; four legged (quadruped) or snake like structures are also there. In this section we will review the mathematical models proposed and developed to study biological CPGs including different types of animal locomotion [5].

The movements are generated in vertebrates by oscillator circuits. These oscillatory units are the basic elements through which different type of movements are generated, called CPG circuits.

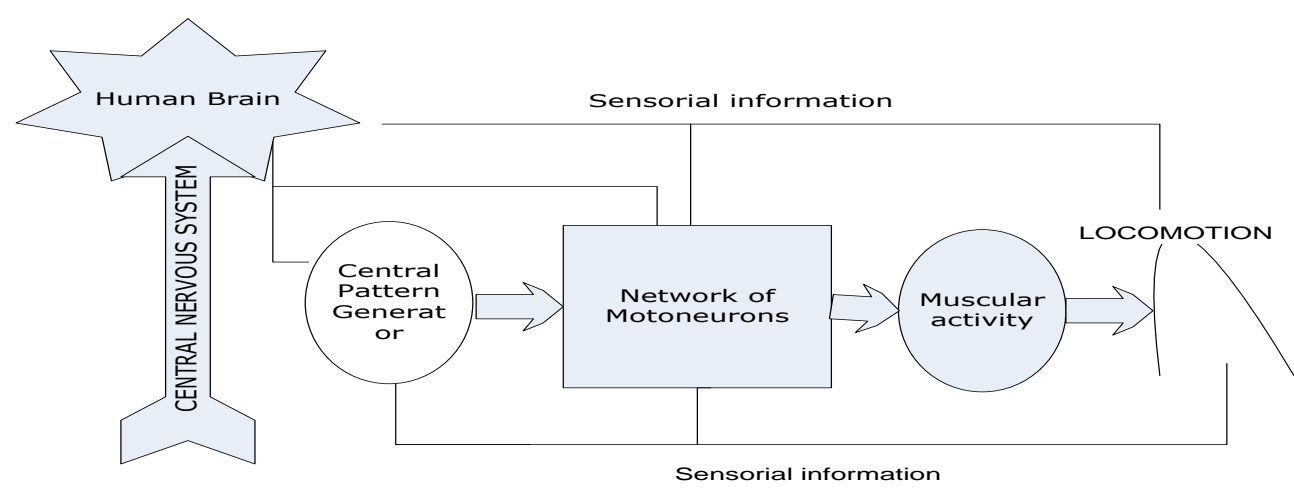

Figure-1 Control system of the human locomotion. 
The CPG circuits consists of some neurons and found in the spinal cord of vertebrate animals. Theses CPG circuits consists of some neurons and found in the spinal cord of vertebrate animals. These CPG circuits are responsible for periodic movement like trotting, swimming, walking, flying and running etc.

The Matsuoka neural oscillator is generally used by the authors very frequently in most of the papers. The idea of Matsuoka neural oscillator is that it consists of two mutually inhibited neurons which suppress the activity of one another in order to generate oscillation in the oscillatory unit.

The mathematical equations of the Matsuoka neural oscillator described by using the following differential equations [6]

$$
T\left(d u_{1} / d t\right)=-u_{1}-w y_{2}-b v_{1}+u_{0}
$$

$T\left(d u_{2} / d t\right)=-u_{2}-w y_{1}-b v_{2}+u_{0}$

$$
\begin{gathered}
T^{\prime}\left(d v_{1} / d t\right)=-v_{1}+y_{1} \\
T^{\prime}\left(d v_{2} / d t=-v_{2}+y_{2}\right.
\end{gathered}
$$

$y_{i}=f\left(u_{i}\right), f\left(u_{i}\right)=\max \left(0, u_{i}\right)$ for $i=1,2$

Where $u_{i}$ is the inner state of the ith neuron; $y_{i}$ is the output of the ith neuron; $\mathrm{v}_{\mathrm{i}}$ is a variable representing the degree of the adaptation or self-inhibition effect of the ith neuron; $u 0$ is an external input with a constant rate; $\mathrm{w}$ is a connecting weight; and $\mathrm{T}$ and $\mathrm{T}$ ' are time constants of the inner state and the adaptation effect, respectively. Other oscillators used by researchers are Vanderpol oscillators, Rayleigh oscillators etc.

The Vanderpol oscillator is a non- conservative oscillator with non - linear damping. It evolves in time according to the second order differential equation [2].

$$
\frac{d_{2} x}{d t^{2}}-m\left(\frac{1}{x^{2}}\right) \frac{d x}{d t}+x=0
$$

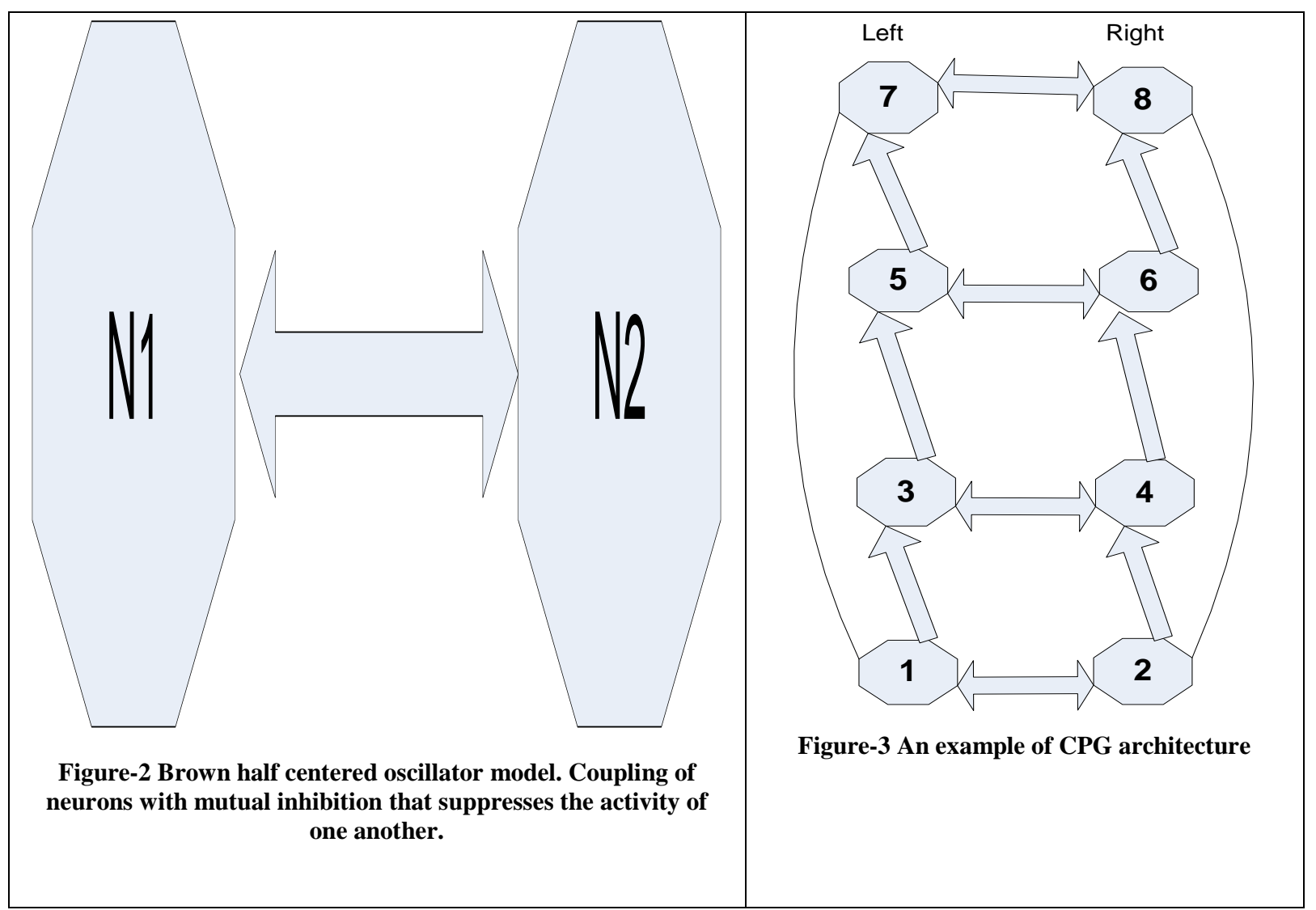


In Figure-3 the design methodology follows a biologically inspired approach to design the controller for a humanoid. The given model is based on the observation of the gait patterns of animals and humans. For the humans the stance phase is the

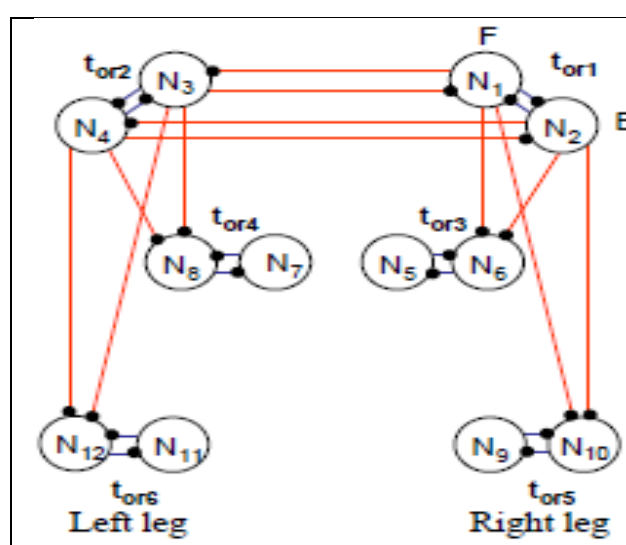

phase during which the limb touches the ground, in swing phase the limb lifts off the ground. However most of the CPG models based on coupled oscillators are not able to separate the swing and stance phase durations [6].

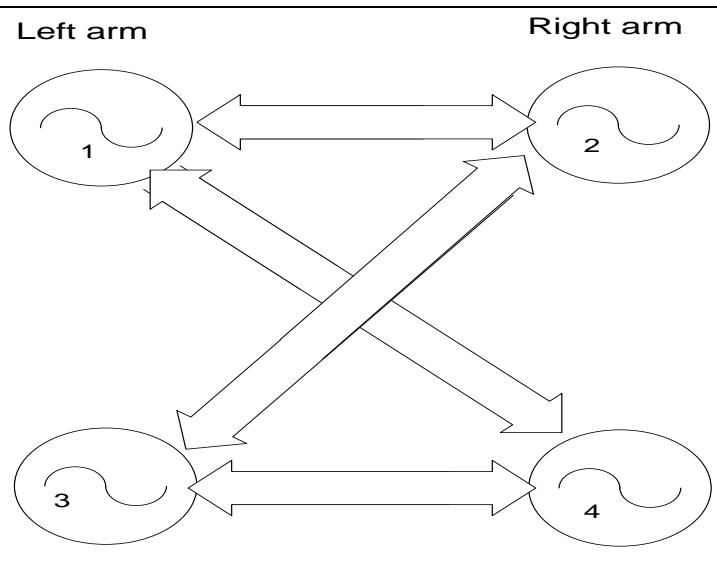

Left leg

Right leg

Figure 4 - another examples of CPG architectures consisting eight or more neural oscillators

The diagram shows that a CPG network consists of several neural oscillators for hip, knee and ankle. The oscillator activates other oscillators and communicates with one another in a specified fashion.

\section{MACHINE LEARNING TECHNIQUES FOR CPG DESIGN IN BIPEDS}

In future a robot is not only constrained to an engineer or some people who are engaged in research and development of robots but also in almost all fields about which a human being can think or imagine [7].

The medical field, agricultural field, production in industries or factories, academics and a number of non-technical fields. So in such a diverse situation if we have some mechanism through which a non-technical person may trained the robot according to its need in an efficient, easy and natural manner is a challenging work and directly related to the concept of machine learning which is very popular technique in current scenario of technological advancement [8].

Some techniques of machine learning like Deep learning and Reinforcement learning are not so competent due to some limitations of these techniques like Deep learning requires large datasets to train the network whereas Reinforcement learning uses substantial and expensive examination to accumulate data points used for learning [9].
Table 1. Use of Machine learning techniques from 2010 onwards in Humanoid Robotics

\begin{tabular}{|c|c|c|c|c|}
\hline S.No. & Year & $\begin{array}{c}\text { Total } \\
\text { Paper } \\
\text { reviewed }\end{array}$ & $\begin{array}{c}\text { Use of } \\
\text { Machine } \\
\text { Learning } \\
\text { techniques } \\
\text { (in no.s) }\end{array}$ & $\begin{array}{c}\text { Use of } \\
\text { Machine } \\
\text { Learning } \\
\text { techniques } \\
\text { (in \%) }\end{array}$ \\
\hline 1 & 2010 & 33 & 4 & $12.12 \%$ \\
\hline 2 & 2011 & 37 & 7 & $18.91 \%$ \\
\hline 3 & 2012 & 35 & 5 & $14.28 \%$ \\
\hline 4 & 2013 & 34 & 6 & $17.64 \%$ \\
\hline 5 & 2014 & 41 & 9 & $21.95 \%$ \\
\hline 6 & 2015 & 30 & 5 & $16.66 \%$ \\
\hline 7 & 2016 & 37 & 9 & $\mathbf{2 4 . 3 2 \%}$ \\
\hline 8 & 2017 & 30 & 9 & $\mathbf{3 0 . 0 0 \%}$ \\
\hline 9 & 2018 & 32 & 11 & $\mathbf{3 4 . 3 7 \%}$ \\
\hline
\end{tabular}

Source: International Journal of Humanoid Robotics, World scientific pub. Company Pvt. Ltd.

The Table 1 and Figure 5 and 6 given, clearly show the growth of machine learning techniques used now a day to design the different aspects of humanoid robots. From 2016 onwards this is high in comparison to the average and previous years.

Another approach called Interactive machine learning solves the challenges encountered in previous techniques. In this method the human being is the part of the learning process by providing the truth values of the environment during exploration of the mechanism. The human teacher also control the mechanism in such a way that it cannot harm the trainer or any other thing exists in the environment during learning process and after learning process is over [10-13]. 


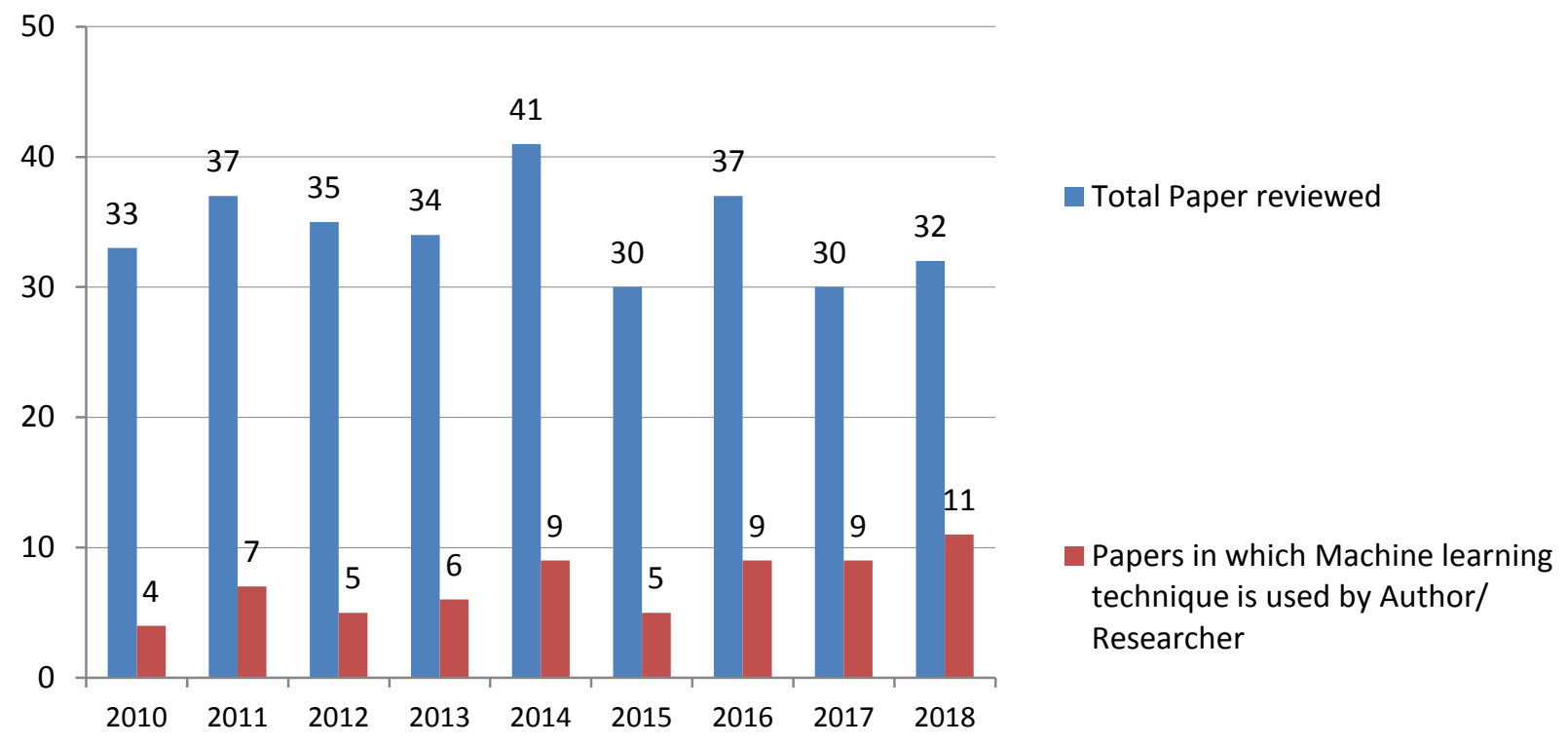

Figure-5 Graph between ML techniques used and total paper reviewed

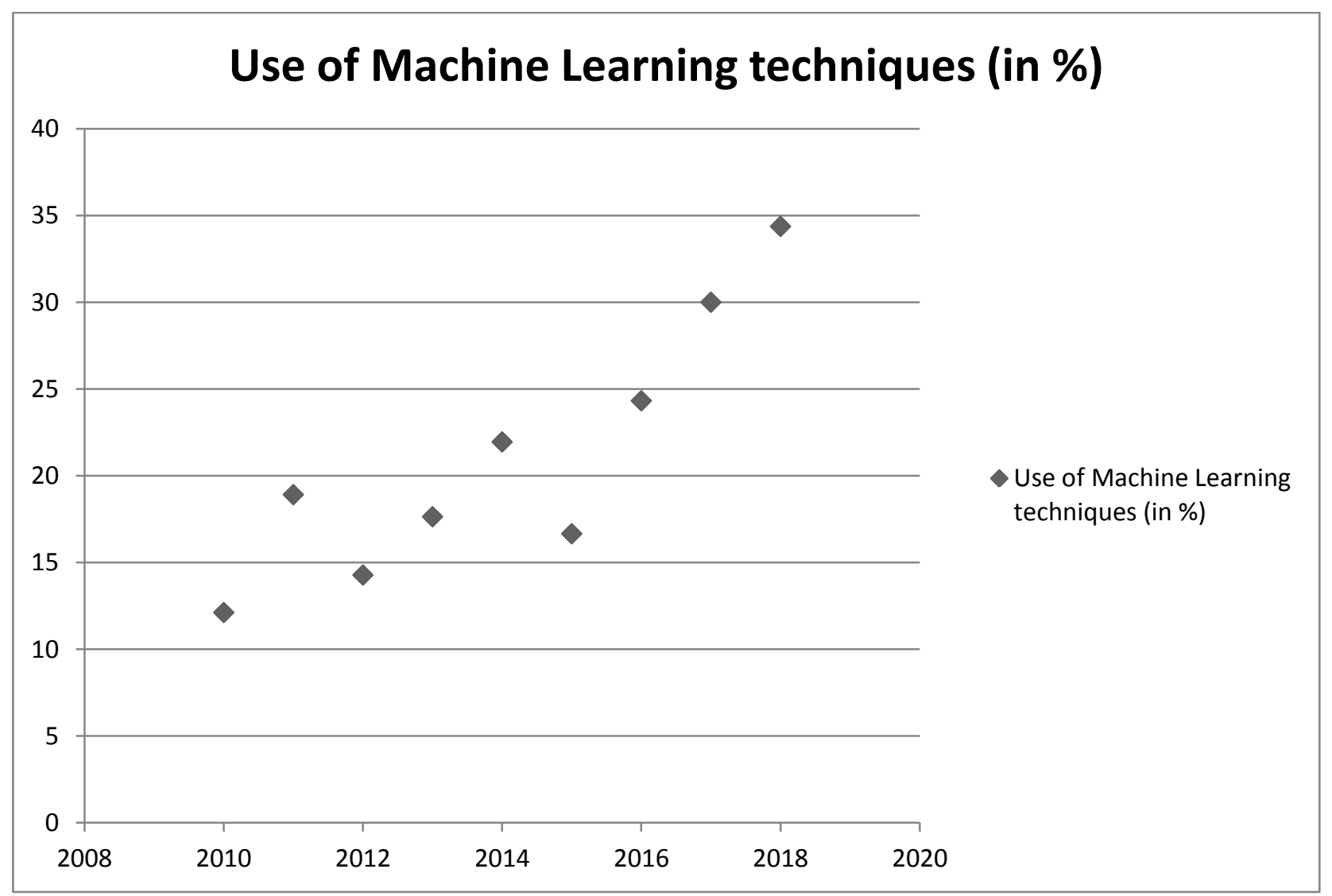

Figure-6 Graph shows the increasing use of Machine Learning techniques in Humanoid Robotics 


\section{DISCUSSION AND CONCLUSION}

Researchers however propose a number of oscillators based models for CPG network and prove the capability of their model by using different tools and techniques.But every model has its specified capability or limitations. We cannot say exactly that a particular model works efficiently in almost all the situations. And till now we have noticed that there is no systematic method of parameter tuning, so it's a challenge for the future researchers to derive a systematic method for parameter tuning in order to achieve the stable locomotion of the humanoids.
We have noticed from last few years that machine learning techniques such as supervised learning which includes neural networks, support vector machines, decision trees and Unsupervised learning, Reinforcement learning are very frequently used by the researcher for modeling different aspects of the humanoid robots. So it is clear that the future is devoted to the machine learning to learn and model various aspect of the real world too. The detailed methods upto some depth of machine learning is given in Table 2 .

Table 2. Different Machine learning techniques and their pros and cons

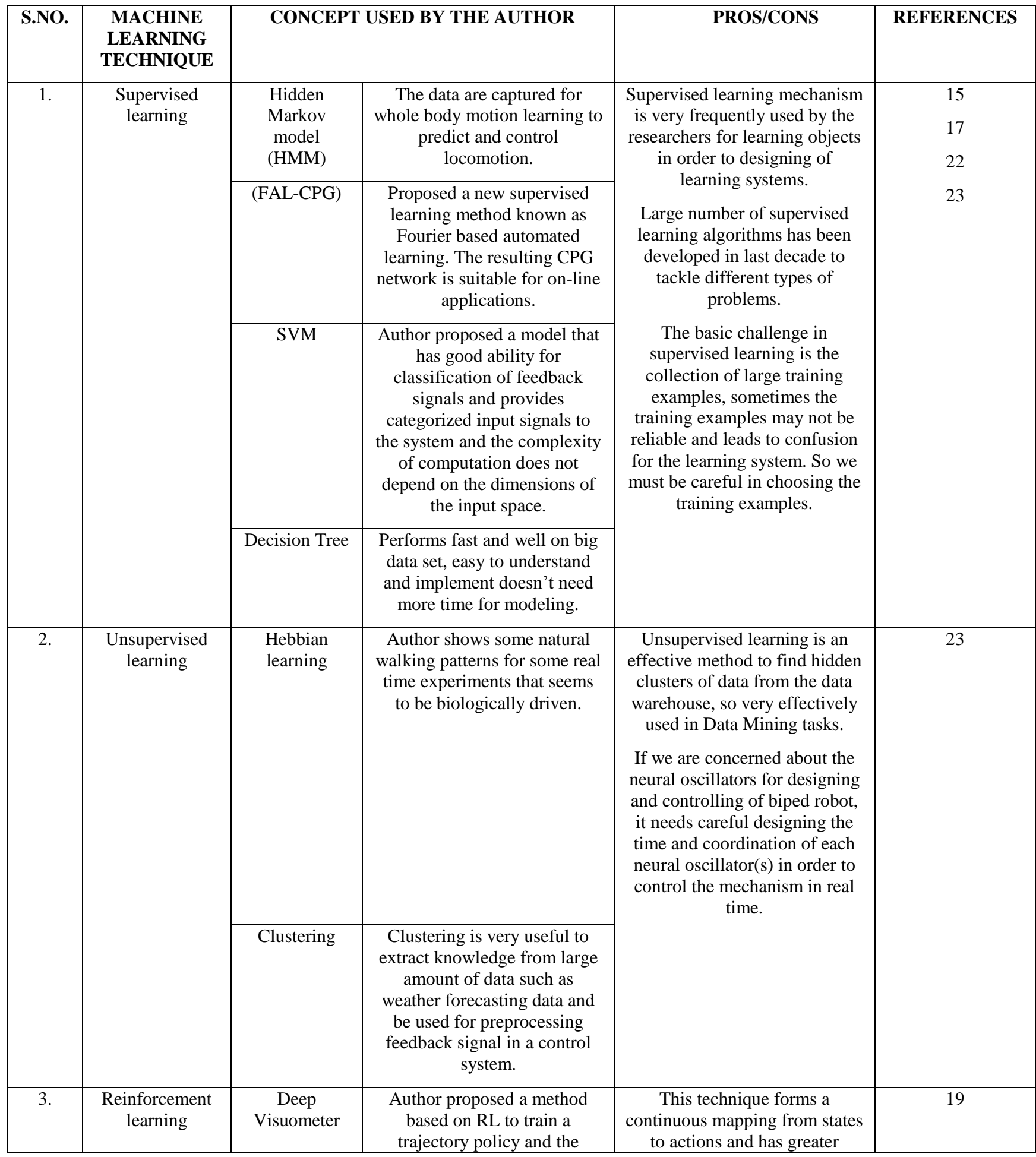




\begin{tabular}{|c|c|c|c|c|c|}
\hline & & & $\begin{array}{l}\text { torque generation signals at } \\
\text { robot arms. }\end{array}$ & \multirow{2}{*}{$\begin{array}{l}\text { capability to learn high } \\
\text { dimensional continuous spaces } \\
\text { easy to understand, tune } \\
\text { learning parameters, requires } \\
\text { low computation and fast } \\
\text { convergence. }\end{array}$} & \multirow[t]{2}{*}{14} \\
\hline & & $\begin{array}{l}\text { Actor- critic } \\
\text { Q-learning }\end{array}$ & $\begin{array}{l}\text { In Reinforcement learning the } \\
\text { training process is somehow } \\
\text { complex since some functions } \\
\text { are required to be adjusted } \\
\text { properly and the learning rate } \\
\text { is generally low. }\end{array}$ & & \\
\hline \multirow[t]{2}{*}{4.} & Deep learning & $\begin{array}{c}\text { Adaboost } \\
\text { classification }\end{array}$ & $\begin{array}{l}\text { Author use a boosting } \\
\text { classifier and week learners } \\
\text { for object detection and } \\
\text { tracking in real time. As a } \\
\text { future the author suggests the } \\
\text { comparison of existing } \\
\text { classifiers to machine } \\
\text { learning techniques such as } \\
\text { deep learning. }\end{array}$ & \multirow{2}{*}{$\begin{array}{l}\text { The rate of detection of objects } \\
\text { is dramatically improved by } \\
\text { using such techniques. } \\
\text { Such systems require large } \\
\text { databases to produce efficient } \\
\text { and competent classifiers. The } \\
\text { time to process and analysis is } \\
\text { not so competent. } \\
\text { Multiple degrees of freedom } \\
\text { may produce challenges } \\
\text { however. }\end{array}$} & \multirow[t]{2}{*}{$\begin{array}{l}16 \\
18\end{array}$} \\
\hline & & $\begin{array}{l}\text { Two phase } \\
\text { learning } \\
\text { model }\end{array}$ & $\begin{array}{l}\text { The method is useful for } \\
\text { production industry } \\
\text { specifically for multi variety } \\
\text { product development. }\end{array}$ & & \\
\hline 5. & $\begin{array}{l}\text { Iterative } \\
\text { learning }\end{array}$ & $\begin{array}{l}\text { Integration of } \\
\text { controllers } \\
\text { using Fuzzy } \\
\text { logic }\end{array}$ & $\begin{array}{l}\text { Author proposed a fuzzy } \\
\text { iterative learning control } \\
\text { strategy for position tracking } \\
\text { problem of powered ankle } \\
\text { prosthesis. }\end{array}$ & $\begin{array}{l}\text { This concept of power ankle } \\
\text { prosthesis is especially useful } \\
\text { for disabled people. } \\
\text { To repeat powered ankle } \\
\text { prosthesis in specified time is a } \\
\text { challenging task however. }\end{array}$ & 21 \\
\hline
\end{tabular}

\section{REFERENCES}

[1] Taga, G. A model of the neuro-musculo-skeletal system for human locomotion. i. emergence of basic gait. Biological Cybernetics, Vol. 73, pp. 97-111, 1995.

[2] Auke Jan Ijspeert. Central pattern generators for locomotion control in animals and robots: A review. ELSEVIER Journal on Neural Networks, Vol. 21, pp. 642-653, 2008

[3] Taga, G. A model of the neuro-musculo-skeletal system for human locomotion. \& real-time adaptability under various constraints. Biological Cybernetics, Vol. 73, pp. 113-121, 1995.

[4] Matsuoka, K. Mechanisms of frequency and pattern control in the neural rhythm generators. Biol. Cybern, Vol. 56, pp. 345-353, 1987.

[5] Taga, G. A model of the neuron-musculo-skeletal system for anticipatory adjustment of human locomotion during obstacle avoidance. Biological Cybernetics, Vol. 78, pp. 9-17, 1998 .

[6] Taga, G., Yamaguchi, Y., Shimizu, H. Self-organized control of bipedal locomotion by neural oscillators in unpredictable environment. Biol. Cybern, Vol. 65, pp. 147-159, 1991.

[7] Chen-Chien Hsu, Wei-Yen Wang, Tung-Yuan Lin, YinTien Wang and Teng-Wei Huang, Enhanced Simultaneous Localization and Mapping (ESLAM) for
Mobile Robots, International journal of humanoid robotics, Vol. 14, Issue 02, 2017.

[8] Takeshi U, Toshiharu H, Katsuji U. Evolution strategies for bipedal locomotion learning using nonlinear oscillators. Osaka University, Japan, AUG 2010.

[9] K. Noda, H. Arie, Y. Suga, and T. Ogata. Multimodel integration learning of robot behavior using deep neural networks. Robot autonomous systems, Vol-62, no. 6, p.p. 721-736, 2014

[10] Manoj S. and Andy Ruina, Computer Optimization of a minimal biped model discovers walking and running, Biorobotics and Locomotion Laboratory, Cornell University, Ithaca, NY USA, 2006 Nature.

[11] Guang Lei Liu, Maki K. Habib, Keigo Watanabe, and Kiyotaka Izumi, The Design of Central Pattern Generators based on the Matsuoka Oscillator to Generate Rhythmic Human Like Movement for Biped Robots, Saga University Honjomachi, Saga 840-8502, Japan, May 2007.

[12] Lingling Chen, Peng Yang, He Chen, Xi Guo, Gait optimization of Biped Robot Based on Mix Encoding Genetic Algorithm, School of Electrical Engineering and Automation, Hebei University of Technology, Tianjin, China, IEEE 2007.

[13] PanduRangaVundavilli, Dilip Kumar Pratihar, Soft computing based gait planners for a dynamically 
balanced biped robot negotiating sloping surfaces, IIT Kharagpur, India, 2008 ELSEVIER.

[14] Slawomir Grzonka, Andreas Karwath, Frederic Dijoux, and Wolfram Burgard, Activity-Based Estimation of Human Trajectories, IEEE Transactions on robotics , 2011

[15] J.Kim, Naveen Kumar,Vikas Panwar, J.H. Borm and J.Chai; Adaptive neural controller for visual servoing of Robot Manipulators with camera-in-hand configuration, Journal of mechanical science and Technology, Vol. 28, No. 8, pp 2313-2323, 2012

[16] Emmanuel senft, Paul Baxter, James Kennedy, Supervised autonomy for online learning in human robot interaction, Elsevier pattern recognition letters, March 31,2017 .

[17] Kai hu, Dongheui Lee, Bipedal locomotion primitive learning, Control and prediction from human data, $10^{\text {th }}$ IFAC symposium on robot control, September 5-7, 2012

[18] Sidahmed Benabderrahmane, Combining boosting machine learning and swarm intelligence for real

[19] time object detection and tracking: towards new meta heuristics boosting classifiers, Springer Nature, November 22, 2017

[20] S. Levine et al, "End to End training of deep visuometer policies " Journal of Machine Learning, vol-17, no.35 Apr 2016.

[21] Kaiyang Yin, Muye Pang, Kui Xiang, Jing Chen, Shenpei Zhou, "Fuzzy iterative learning control starategy for powered ankle prosthesis", Springer Nature Sigapore Pte. Ltd. March 2018

[22] J.P. Ferreira, M.crisostomo, A.P. Coimbra, B.Ribeiro, Simulation control of a biped robot with support vector regression, Proceeding IEEE Int. Symp. Int. signal process, 2007, p.p. 1-7

[23] Shouyi Wang et al., Machine Learning Algorithms in Bipedal Robot Control, IEEE TRANSACTIONS ON SYSTEMS, MAN AND CYBERNETICS, VOL-42, NO.-5, SEPTEMBER 2012

[24] Min-Su Kim and Jun Ho Oh, Posture control of a Humanoid Robot with a compliant ankle joint, International journal of humanoid robotics, Vol. 07, No. 01, p.p. 5-29, 2010.

[25] Hisashi Sugiura et al., Reactive self collision avoidance with dynamic task prioritization for humanoid robots, International journal of humanoid robotics, Vol. 07, No. 01, p.p. 31-54, 2010

[26] Q.WU and J.CHEN, Effects of ramp angle and mass distributions on passive dynamic gait - An experimental study, International journal of humanoid robotics, Vol. 07, No. 01, p.p. 55-72, 2010.

[27] J.G.Daniel Kassen and Martijn Wisse, Fall detection of two legged walking robots using multi-way principal component analysis, International journal of humanoid robotics, Vol. 07, No. 01, p.p. 73-93, 2010

[28] Qirong Mao, Xioajia Wang, Yongzhao Zhan, Speech emotion recognition method based on improved decision tree and layered feature selection, International journal of humanoid robotics, Vol. 07, No. 02, p.p. 245-261, 2010
[29] Armin Hornung, Stefan Obwald, Daniel Maier and Maren Bennewitz, Monte Carlo Localization for Humanoid Robot Navigation in Complex Indoor Environments, International journal of humanoid robotics, Vol. 11, Issue 02 (2014), 1441002

[30] Toshiya Nishi and Tomomichi Sugihara, Motion planning of a Humanoid Robot in a complex environment using RRT and Spatiotemporal PostProcessing Techniques, International journal of humanoid robotics, Vol. 11, Issue 02 (2014), 1441003

[31] Said G. Khan, Guido Herrmann, Mubarak Al Grafi, Tony Pipe and Chris Melhuish, Compliance control and Human Robot Interaction: Part 1 - Survey, International journal of humanoid robotics, Vol. 11, Issue 03 (2014), 1430001

[32] Said G. Khan, Guido Herrmann, Mubarak Al Grafi, Tony Pipe and Chris Melhuish, Compliance control and Human Robot Interaction: Part II - Experimental Examples, International journal of humanoid robotics, Vol. 11, Issue 03 (2014), 1430002

[33] Javier Moya, Javier Ruiz-del-Solar, Marcos Orchard and Isao Parra-Tsunekawa, Fall Detection and Damage Reduction in Biped Humanoid Robots, International journal of humanoid robotics, Vol. 12, Issue 01 (2015), 155001

[34] Martin D. Cooney, Shuichi Nishio and Hiroshi Ishiguro, Importance of Touch for Conveying Affection in a multimodal Interaction with a small Humanoid Robot, International journal of humanoid robotics, Vol. 12, Issue 01 (2015), 155002

[35] Yeoun-Jae Kim, Joon-Yong Lee and Ju-Jang Lee, A Torso moving Balance Control Strategy for a walking Biped Robot Subject to External Continuous Forces, International journal of humanoid robotics, Vol. 12, Issue 01 (2015), 155003

[36] Jonathan Spitz, Eric Sidorov and Miriam Zacksenhouse, Humanoids can take advantages of Crab-Walking Gaits, International journal of humanoid robotics, Vol. 12, Issue 01 (2015), 155004

[37] Wenzhen Yang, Xinli Wu and Hua Zhang, Workspace Modeling and Analysis for Dexterous Hands, International journal of humanoid robotics, Vol. 12, Issue 01 (2015), 155006

[38] Jan Kedzierski, Pawel Kaczmarek, Michal Dziergwa and Krzysztof Tchon, Design for a Robotic Companion, International journal of humanoid robotics, Vol. 12, Issue 01 (2015), 155007

[39] Francisco Martin, Carlos E. Aguero and Jose M. Canas, Active Visual Perception for Humanoid Robots, International journal of humanoid robotics, Vol. 12, Issue 01 (2015), 155009

[40] Sung Taek Cho and Seul Jung, Combining two Control Techniques for the Fast Movement of a Two-Wheel Mobile Robot, International journal of humanoid robotics, Vol. 12, Issue 02 (2015), 1550020

[41] Yeoun-Jae Kim, Joon-Yong Lee and Ju-Jang Lee, A Balance control strategy for a Walking Biped Robot under Unknown Lateral External Force using a Genetic 
Algorithm, International journal of humanoid robotics, Vol. 12, Issue 02 (2015), 1550021

[42] Rok Vuga, Bojan Nemec and Ales Ude, Enhanced policy Adaptation Through Directed Explorative Learning, International journal of humanoid robotics, Vol. 12, Issue 03 (2015), 1550028

[43] Jemin Hwangbo, Christian Gehring, Hannes Sommer, Roland Siegwart and Jonas Buchli, Policy learning with an Efficient Black-Box Optimization Algorithm, , International journal of humanoid robotics, Vol. 12, Issue 03 (2015), 1550029

[44] Akihiko Yamaguchi, Christopher G. Atkeson and Tsukasa Ogasawara, Pouring skills and Learning Modeled from Human Demonstrations, , International journal of humanoid robotics, Vol. 12, Issue 03 (2015), 1550030

[45] Peter Kaiser, Nikolaus VahrenKamp, Fabian Schultje, Julia Borras and Tamim Asfour, Extraction of WholeBody Affordances for Loco-Manipulation Tasks, , International journal of humanoid robotics, Vol. 12, Issue 03 (2015), 1550031

[46] Olivier Hugues, Vincent Weistroffer, Alexis Paljic, Philippe Fuchs, Ahmad Abdul Karim, Thibaut Gaudin and Axel Buendia, Determining the important subjective criteria in the Perception of Human like Robot Movements Using Virtual Reality, International journal of humanoid robotics, Vol. 13, Issue 02 (2016), 1550033

[47] Roberta AIo and Giacomo Mantriota, Optimal Grip Points with Human Hand, International journal of humanoid robotics, Vol. 13, Issue 02 (2016), 1550036

[48] Juan Alejandro Castano Zhibin Li, Chengxu Zhou, Nikos Tsagarakis and Darwin Caldwell, Dynamic and Reative Walking for Humanoid Robots Based on Foot Placement Control, International journal of humanoid robotics, Vol. 13, Issue 02 (2016), 1550041

[49] Meteb M. Altaf, Bassant M. Elbagoury, Fahad Alraddady and Mohamed Roushdy, Extended CaseBased Behavior Control for Multi-Humanoid Robots, International journal of humanoid robotics, Vol. 13, Issue 02 (2016), 1550035

[50] Kai Xu, Huan Liu, Yuheng Du and Xiangyang Zhu, A Comparative study for Postural Synergy Synthesis Using Linear and Nonlinear Methods, International journal of humanoid robotics, Vol. 13, Issue 03 (2016), 1650009
[51] Takeshi Nishida, Yuki Okatani and Kenjiro Tadakuma, Development of Universal Robot Gripper Using MR $\alpha$ Fluid, , International journal of humanoid robotics, Vol. 13, Issue 04 (2016), 1650017

[52] Jumpei Arata, Kazuo Kiguchi, Masashi Hattori, Masamichi Sakaguchi, Ryu Nakadate, Susumu Oguri and Makoto Hashizume, A Microsurgical Robotic System that Induces a Multisensory Illusion, , International journal of humanoid robotics, Vol. 13, Issue 04 (2016), 1650018

[53] Sahab Omran, Sophie Sakka and Yannick Aoustin, Effects of COM Vertical Oscillation on Joint Torques During 3D Walking of Humanoid Robots, , International journal of humanoid robotics, Vol. 13, Issue 04 (2016), 1650019

[54] Daniele Cafolla and Macro Ceccarelli, Design and Simulation of a Cable-Driven Vertebra-Based Humanoid Torso, , International journal of humanoid robotics, Vol. 13, Issue 04 (2016), 1650015

[55] Jing Zhao and Yuan Wei, A Novel Algorithm of HumanLike Motion Planning for Robotic Arms, International journal of humanoid robotics, Vol. 14, Issue 01 (2017), 1650023

[56] Wenjun Ye, Zhijun Li, Chenguang Yang, Fei Chen and Chun-Yi Su, Motion Detection Enhanced Control of an Upper Limb Exoskeleton Robot for Rehabilitation Training, International journal of humanoid robotics, Vol. 14, Issue 01 (2017), 1650031

[57] Wenzhen Yang, Xinli Wu and Shiguang Yu, A MasterSlave Control Method for Dexterous Hands with Shaking Elimination Strategy, International journal of humanoid robotics, Vol. 14, Issue 01 (2017), 1650016

[58] Li Chen, Wang-Rim Choi, Jeong-Gu Lee, Yi-Gon Kim, Hong-Sik Moon and Young-Chul Bae, Oil-Tank Weld Detection Using EMAT, International journal of humanoid robotics, Vol. 14, Issue 02 (2017), 1750008

[59] Howon Lee and Jangmyung Lee, Optimal Control of an Inverted Ball-Driving Robot Based upon Slip Patterns, International journal of humanoid robotics, Vol. 15, Issue 02 (2018), 1850007

[60] Daniel Herrera, Flavio Roberti, Ricardo Carelli, Victor Andaluz, Jose Varela, Jessica Ortiz and Paul Canseco, Modeling and Path-Following Control of a Wheelchair in Human-Shared Environments, International journal of humanoid robotics, Vol. 15, Issue 02 (2018), 1850010 